\title{
Management based on grading of animation deformity following implant-based subpectoral breast reconstruction
}

\author{
Raghavan Vidya ${ }^{1}$, Habib Tafazal ${ }^{2}$, Fathi Salem ${ }^{1}$, Fahad Mujtaba Iqbal ${ }^{3}$, Tapan Sircar ${ }^{1}$ \\ ${ }^{1}$ Department of Breast Surgery, Royal Wolverhampton Hospitals NHS Trust, Wolverhampton; ${ }^{2}$ Department of Breast Surgery, Queen \\ Elizabeth Hospital Birmingham, Birmingham; ${ }^{3}$ St George's Hospital, London, UK
}

Subpectoral breast reconstruction using implants and meshes have been used widely in Europe, the United States and the United Kingdom. Although this technique has several advantages, animation deformity is a well-documented problem. We propose a new grading system to classify breast animation in patients undergoing subpectoral implant based breast reconstruction. We also discuss different techniques to avoid and correct animation deformity.

Keywords Animation / Breast / Mammaplasty / Breast implants
Correspondence: Raghavan Vidya Department of Breast Surgery, Royal Wolverhampton Hospitals NHS Trust, New Cross Hospital, Wolverhampton, WV10 0QP, UK

Tel: +441902-695969

Fax: +441902-695754

E-mail: Raghavan.Vidya@nhs.net

We wish to acknowledge Prof John Kim, Northwestern Memorial Hospital, Chicago, USA for his guidance.

This article contains Supplemental Videos S1 and S2.

Received: 30 Jul 2017 • Revised: 6 Sep 2017 • Accepted: 12 Sep 2017

pISSN: 2234-6163 • elSSN: 2234-6171 • https://doi.org/10.5999/aps.2017.01242 • Arch Plast Surg 2018;45:185-190

\section{INTRODUCTION}

Breast cancer is the commonest cancer in women, with over 50,000 new cases diagnosed each year in the United Kingdom. Surgery remains the commonest treatment modality. There is an increasing trend of skin sparing mastectomies with implantbased breast reconstruction for therapeutic treatment of breast cancer and for risk-reducing surgery in high-risk patients. Conventionally, a sub-pectoral implant placement with partial detachment of the pectoralis major muscle is recommended, but this can be associated with partial muscle injury resulting in impaired function, animation deformity, and increased postoperative pain [1].

Since the introduction of techniques using acellular dermal matrices (ADM), synthetic meshes and autologous dermal slings, implant-based reconstruction is being further favoured. It accounts for $40 \%-60 \%$ of all breast reconstructions performed in the United Kingdom and nearly $75 \%$ in the United States [2,3]. All the techniques for lower pole coverage require the recruitment of pectoralis muscle [4].

\section{IDEA}

\section{Pectoralis major: anatomy and physiology}

The pectoralis major muscle arises proximally from the anterior surface of the medial half of the clavicle (clavicular head) and lateral aspect of the manubrium and body of the sternum, the upper six costal cartilages and the aponeurosis of the external oblique (sternal head). It is inserted into the bicipital groove of the humerus and deltoid tuberosity. It is innervated by the me- 
dial and lateral pectoral nerve, with the clavicular head innervated by $\mathrm{C} 5-\mathrm{C} 6$ and the sternocostal head innervated by $\mathrm{C} 7-\mathrm{T} 1$. The primary function of the pectoralis major is to medially rotate and adduct the humerus, drawing the scapular anterioinferiorly. The muscle has been observed to have a narrow or high distribution in about $72 \%$ of patients [5]. Assessment of its function and its integrity is vital before performing subpectoral based breast reconstruction.

\section{Classic technique}

Subpectoral implant based reconstruction involves the division of the pectoralis major muscle which is then incorporated with the mesh to form a pocket for the implant (Fig. 1). The mesh with the muscle forms an internal bra or hammock to hold the definitive implant. The advantages include improved lower pole projection, single stage reconstruction, and a better psychological outcome [6]. However, when the muscle is detached to form a pocket for the implant, the muscle with the implant forms one unit and movements causing contraction of the muscle can cause displacement of the implant (Fig. 1). This is known as "animation deformity" and has been well identified as a problematic entity [1]. It can also be postulated that the degree of animation is influenced by the biomechanical consequence of muscle disconnection and contraction from the chest particularly in relation to adhesion of the muscle to the parenchyma and the overlaying skin.

\section{Fig. 1. Subpectoral reconstruction and breast animation}

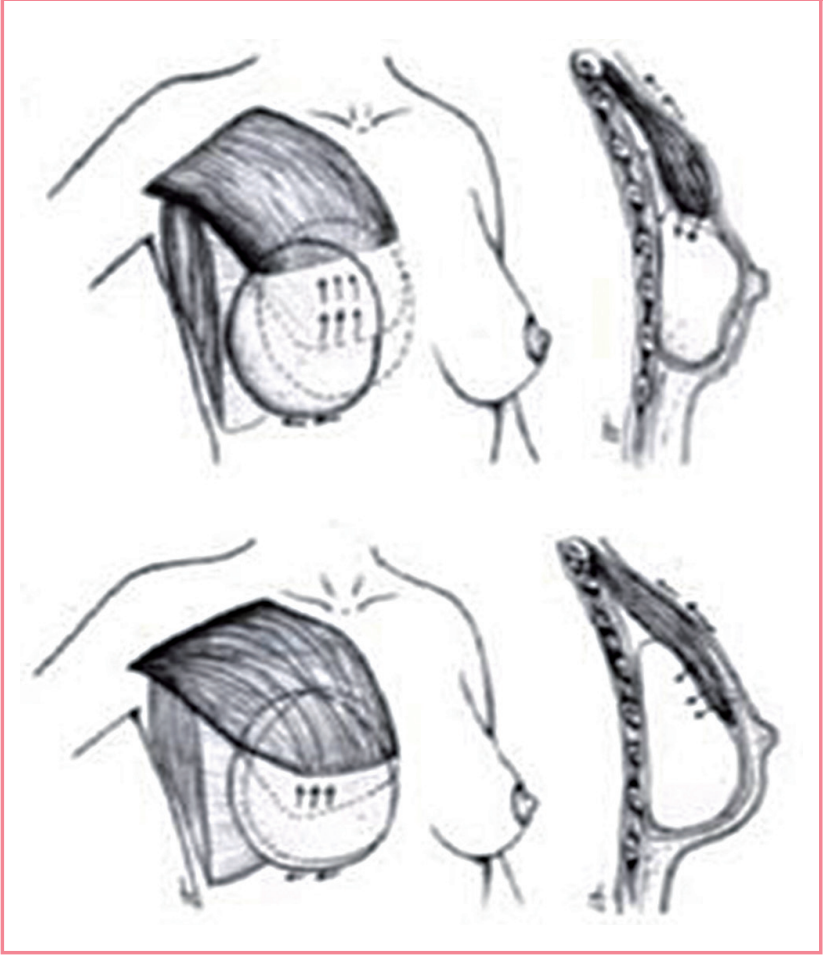

\section{Animation}

Animation was originally described in subpectoral breast augmentation and can be sub-classified as deformity or distortion of the breast. This occurs with arm movement and contraction of the muscle [7]. Animation deformity has been observed in more than $50 \%$ of patients who underwent subpectoral breast augmentation, most noticeable following activity (lifting and heavy exercises). In 2009, a survey was circulated to 195 patients; of the 69 responses, only $22.5 \%(n=9)$ reported no distortion with primary subpectoral breast augmentation with $15 \%$ of patients reporting moderate to severe deformity. It should be noted, however, that the response rate was poor (35\%) [8].

\section{Anatomic rationale}

Subpectoral implant based reconstruction involves the division of the pectoralis major muscle to form a pocket for the implant. The pectoralis muscle is partly or completely divided from its origin and the mesh is secured to its free lower edge. Deformity occurs during normal activity and exercise, which involves flexion, adduction and medial rotation of the arm. This is because the pectoralis muscle contracts and the subpectoral implant is forced to move from its normal position as one unit. Furthermore, the adhesion of the mastectomy flap (skin) and the degree of retraction of the pectoralis muscle (roller blind effect) would affect the degree of animation [8]. Therefore, the implant is displaced due to the action of the muscle; the degree of which is dependent on the force of the action (Fig. 1). It can be postulated that the muscle mesh interface with the capsule can distort the breast when the muscle contracts forcing the mesh capsule unit to be displaced. The degree of displacement depends on the force of contraction along with the degree of mesh capsule integration with the muscle. It could also be affected by the degree of muscle detachment as partial or complete muscle detachment can cause minimum visible or extreme distortion.

With the increase in "implant animation," we feel that an algorithmic approach, including grading of the deformity and subsequent management is required.

\section{Animation grading}

Grading of breast animation is based on distortion and the movement of the implant on contraction of pectoralis major muscle and interference with activity (Table 1, Figs. 2-4, Supplemental Videos S1, S2).

We define normal activity as dressing, cleaning, cooking, and writing; and exercise activity as gym exercises, swimming, and cycling. 


\section{DISCUSSION}

The classic subpectoral breast reconstruction offers reasonable

\begin{tabular}{|c|c|c|}
\hline Grade & Definition & Management \\
\hline 1 & $\begin{array}{l}\text { No visible distortion and displacement of the implant } \\
\text { during muscle contraction, both during normal and } \\
\text { exercise activity }\end{array}$ & No action needed \\
\hline 2 & $\begin{array}{l}\text { Minimal visible distortion with displacement of the } \\
\text { implant (Superolaterally) during muscle contraction } \\
\text { both in normal and exercise activity, grooving may } \\
\text { be seen, unnoticed by patient (Fig. 2) }\end{array}$ & $\begin{array}{l}\text { Offer and discuss } \\
\text { intervention }\end{array}$ \\
\hline 3 & $\begin{array}{l}\text { Moderate visible distortion during muscle contraction, } \\
\text { with displacement of the implant (Superolaterally) } \\
\text { during muscle contraction both during normal and } \\
\text { exercise activity, often noticed by patient (Fig. 3) }\end{array}$ & $\begin{array}{l}\text { Offer and discuss } \\
\text { intervention }\end{array}$ \\
\hline 4 & $\begin{array}{l}\text { Severe distortion during muscle contraction with } \\
\text { persistent displacement of the implant both in } \\
\text { normal and exercise activity, unattractive results } \\
\text { disturbing the patient (Fig. 4) }\end{array}$ & Needs intervention \\
\hline
\end{tabular}

cosmesis but has drawbacks. Although it provides good cover it results in animation deformity with subsequent physiotherapy being frequently required [9].

Subpectoral implant placement with $\mathrm{ADM}$ or various meshes for lower pole reinforcement are used. Other methods of lower pole support have been described such as the use of autogenous dermis as a graft or flap. In the literature it is apparent that animation deformity occurs with any subpectoral breast reconstruction irrespective of the material used for the lower pole reinforcement $[6,9]$.

Hence it is important to grade the animation deformity to assess the severity and address its management. We graded animation based on the degree of deformity, displacement of the implant and the visible distortion during muscle contraction in normal activity or during exercise (Fig. 5). Spear et al. [8] have classified distortion and animation in patients undergoing subpectoral breast augmentation into 3 categories. They conclude that in the setting of augmentation nearly all patients would choose subpectoral positioning of their implants despite some

\section{Fig. 2. A case demonstrating grade 2 animation}

(A) No animation deformity or displacement of the implant seen at rest and (B) with activity minimal visible distortion seen during muscle contraction.
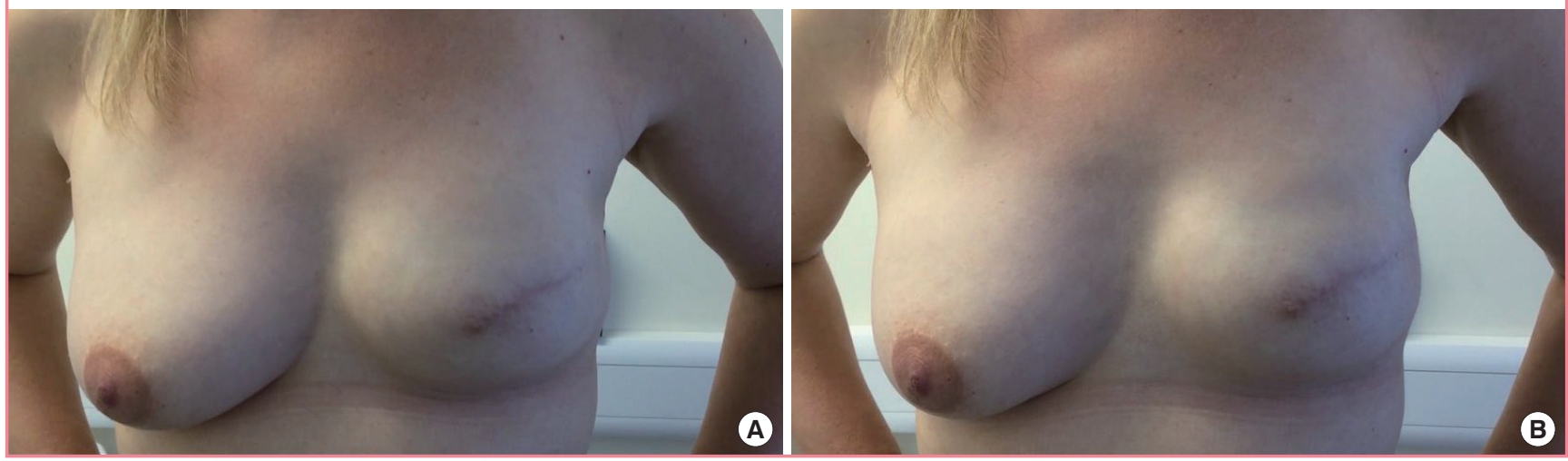

Fig. 3. A case demonstrating grade 3 animation

(A) No animation deformity seen at rest and (B) moderate visible distortion and displacement of implant (superolaterally) seen during muscle contraction.
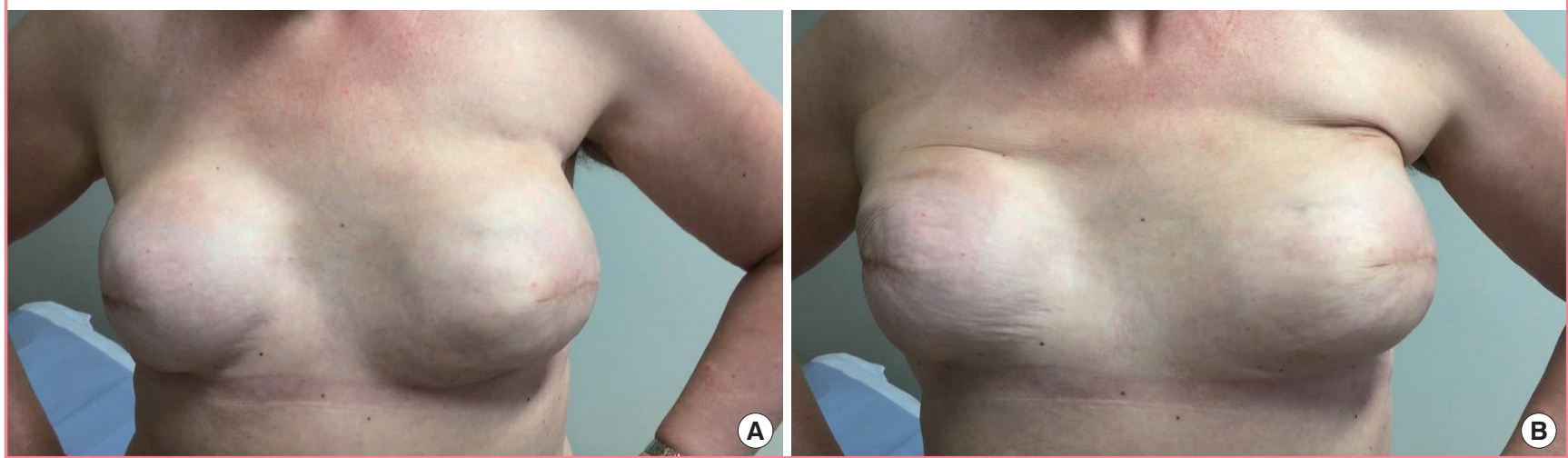


\section{Fig. 4. A patient with grade 4 animation deformity}

(A) Demonstrates severe distortion with unattractive results. (B) Correction of patient with animation deformity using prepectoral technique: 3 months postoperative results.

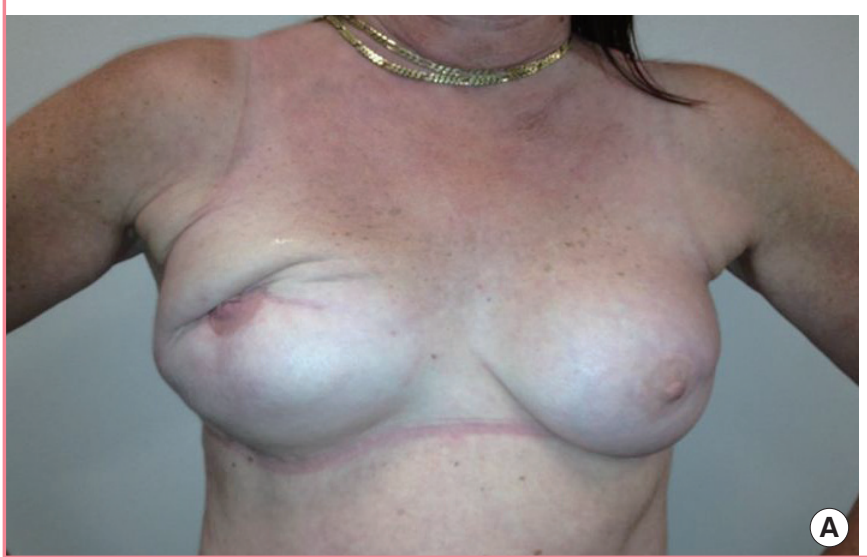

Fig. 5. Selection considerations for the prepectoral technique

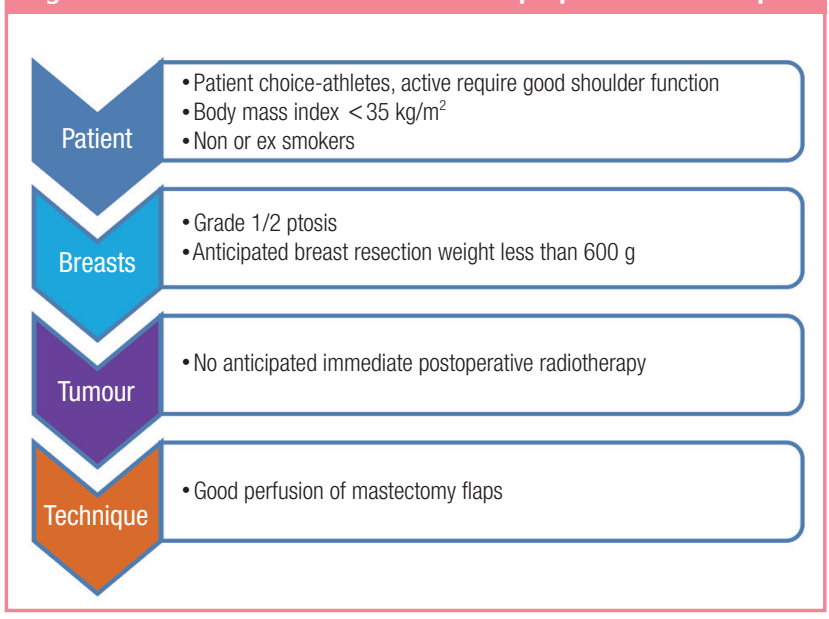

degree of animation. They also agree that patients who may be better candidates for subglandular placement are those for whom exercise is central to their daily living.

Novel techniques of breast reconstruction can be adopted which preserve the pectoralis muscle and avoid animation deformity. Historically, conversion to the prepectoral plane has been difficult due to wrinkling and the thin skin flap demonstrating the implant contour. However, the introduction of meshes has somewhat solved this problem. A variety of meshes are available in the market deriving from both allogenic and xenogenic donor sources. ADMs are the most commonly used meshes and can be derived from human, porcine, or bovine sources. Another form of lower pole coverage involves the use of a dermal sling. This is a useful technique in patients with ptotic breasts. We have briefly described our selection considerations for those undergoing the muscle sparing technique (Fig. 5).

Prepectoral breast reconstruction using a preshaped ADM

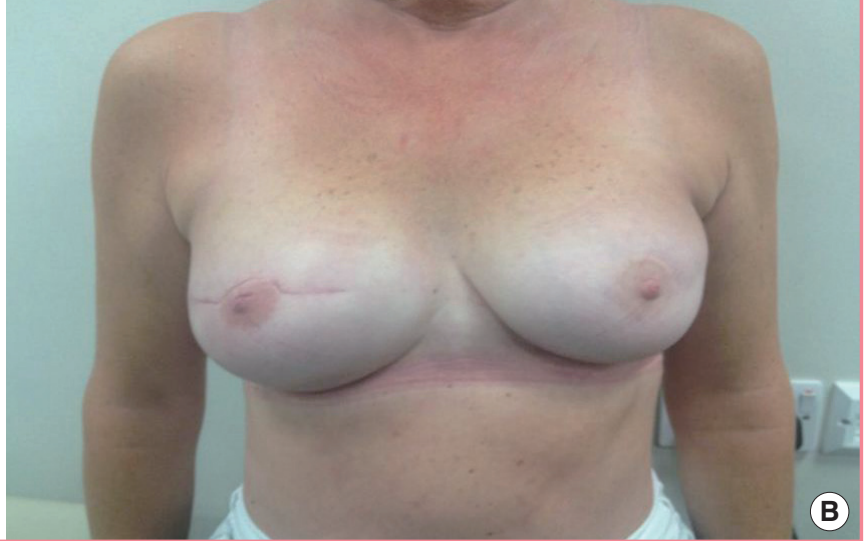

mesh (Braxon manufactured by Decomed, Venice, Italy) has been described. A recent multicentre study of 100 cases using Braxon (median follow-up 17.9 months) was associated with complications including $2 \%$ implant loss, $1 \%$ wound breakdown, 5\% seroma and 2\% haematoma [10]. Animation deformity was completely eliminated using this technique. Other mesh based prepectoral techniques have been described using other ADMs or synthetic meshes like Tiloop. The muscle sparing techniques employ a mesh, which completely wraps the implant, which is placed on the pectoralis muscle and sutured to it, thus anchoring it to the chest wall. The pectoralis major muscle therefore does not need dividing from its attachment and is kept undisturbed. This approach may offer the improved cosmetic outcome that is associated with an ADM sling but without the surgical morbidity associated with creating a subpectoral pocket.

The prepectoral muscle-sparing technique can be considered as a potential alternative to one or two-stage breast reconstruction in patients with moderate size breasts who want to preserve their natural shape. It could be the surgery of choice in athletic patients as it preserves the integrity of the pectoralis major muscle.

The main advantages of the subcutaneous mesh wrapped implant include good cosmesis, reduced muscular pain, and preservation of the pectoralis major muscle. Treatment of subpectoral based implant reconstruction associated with animation deformity includes conversion to the prepectoral plane adoption of techniques, such as lipomodelling or a combination of the two.

Other proposed techniques to prevent muscular animation deformities includes (extrapolated from breast augmentation) release of pectoralis attachments and anterior surface from the breast parenchyma (dual plane), and additional horizontal incisions through the muscle (triple plane) [11]. Subfascial implant 
placement and muscle-splitting approaches have also been proposed to reduce the risk of muscular distortion [12].

In 2014, a retrospective study ( $\mathrm{n}=19)$ was performed on consecutive patients who underwent a change in implant position (subpectoral to the subcutaneous plane) in order to correct severe animation deformity following completed two-stage expander/implant breast reconstruction [13]. This involved access to the pocket through the previous incision followed by division of the junction between the $\mathrm{ADM}$ and pectoralis muscle. The pectoralis was additionally freed from the overlying subcutaneous tissue along a new subcutaneous plane just superficial to the pectoralis fascia; the inferior border of the muscle was sutured to the posterior capsule and chest wall. All 19 patients were noted to have complete animation deformity resolution at follow-up, and 16 of the 19 patients (84.2\%) reported satisfaction at their final follow-up (mean 13.8, range 5-48 months). Complications were seen in 5 breasts (26.3\%); 4 breasts $(21.1 \%)$ requiring capsulectomy and 1 (5.3\%) with seroma. Minor complications (suture erosion, scarring) were noted in 2 breasts (10.5\%). The small sample size and operator bias should be noted and further verification of their work is required, but it does remain a viable alternative to treat excessive breast animation after implant-based breast reconstruction.

Preoperative assessment of patients would determine the suitability for a prepectoral approach to be employed, particularly palpating and pinching the skin to assess the adequacy of the subcutaneous tissue. It is indeed subjective and varies with age, body habitus, previous surgical history, and the patient's tissue quality.

Other treatments including botulinum toxin injections and selective neurectomy of the medial pectoral nerves have also been proposed to prevent the contraction of the pectoralis $[14,15]$. Additional sessions of fat grafting may help conceal the distortion without requiring a formal pocket change.

Despite numerous advances in the techniques and materials used in implant-based breast reconstruction, muscular distortion continues to be a postoperative concern for both the patient and the surgeon. In appropriately selected patients, changing the position of the implant from the subpectoral to the subcutaneous plane and reinforcing the soft-tissue envelope of the breast using $\mathrm{ADM}$ and in some patients, using only fat grafting, the distortion caused by the pectoralis major muscle may be eliminated, with improvement in cosmesis. In appropriately selected patients, a pocket change to a subcutaneous plane is a safe and effective technique for the correction of severe animation deformity following implant-based breast reconstruction.

\section{NOTES}

\section{Conflict of interest}

No potential conflict of interest relevant to this article was reported.

\section{Ethical approval}

The study was approved by the Institutional Review Board of Hospital and performed in accordance with the principles of the Declaration of Helsinki. Written informed consents were obtained.

\section{Patient consent}

The patients provided written informed consent for the publication and the use of their images.

\section{REFERENCES}

1. Reitsamer R, Peintinger F. Prepectoral implant placement and complete coverage with porcine acellular dermal matrix: a new technique for direct-to-implant breast reconstruction after nipple-sparing mastectomy. J Plast Reconstr Aesthet Surg 2015;68:162-7.

2. Jeevan R, Cromwell D, Browne J, et al. The National Mastectomy and Breast Reconstruction Audit 2009: Second Annual Report. A national audit of provision and outcomes of mastectomy and breast reconstruction surgery for women in England [Internet]. Leeds: The NHS Information Centre; c2009 [cited 2017 Sep 30]. Available from: http:// content.digital.nhs.uk/catalogue/PUB02722/clin-audisupp-prog-mast-brea-reco-2009-rep1.pdf

3. American Society of Plastic Surgeons. 2012 Plastic surgery statistics [Internet]. Arlington Heights, IL: American Society of Plastic Surgeons; c2013 [cited 2017 Sep 30]. Available from: https://www.plasticsurgery.org/documents/ News/Statistics/2012/plastic-surgery-statistics-full-report2012.pdf.

4. Gandhi A, Barr L, Johnson R. Bioprosthetics: changing the landscape for breast reconstruction? Eur J Surg Oncol 2013; 39:24-5.

5. Madsen RJ Jr, Chim J, Ang B, et al. Variance in the origin of the pectoralis major muscle: implications for implant-based breast reconstruction. Ann Plast Surg 2015;74:111-3.

6. Sbitany H, Langstein HN. Acellular dermal matrix in primary breast reconstruction. Aesthet Surg J 2011;31(7 Suppl): 30s-37s.

7. Lesavoy MA, Trussler AP, Dickinson BP. Difficulties with subpectoral augmentation mammaplasty and its correction: 
the role of subglandular site change in revision aesthetic breast surgery. Plast Reconstr Surg 2010;125:363-71.

8. Spear SL, Schwartz J, Dayan JH, et al. Outcome assessment of breast distortion following submuscular breast augmentation. Aesthetic Plast Surg 2009;33:44-8.

9. Tebbetts JB. Dual plane breast augmentation: optimizing implant-soft-tissue relationships in a wide range of breast types. Plast Reconstr Surg 2001; 107:1255-72.

10. Vidya R, Masia J, Cawthorn S, et al. Evaluation of the effectiveness of the prepectoral breast reconstruction with Braxon dermal matrix: first multicenter European report on 100 cases. Breast J 2017;23:670-6.

11. Spear SL, Carter ME, Ganz JC. The correction of capsular contracture by conversion to "dual-plane" positioning: tech- nique and outcomes. Plast Reconstr Surg 2006;118:103S$113 S$.

12. Baxter RA. Subfascial breast augmentation: theme and variations. Aesthet Surg J 2005;25:447-53.

13. Hammond DC, Schmitt WP, O'Connor EA. Treatment of breast animation deformity in implant-based reconstruction with pocket change to the subcutaneous position. Plast Reconstr Surg 2015;135:1540-4.

14. Richards A, Ritz M, Donahoe S, et al. Botox for contraction of pectoral muscles. Plast Reconstr Surg 2001;108:270-1.

15. Maxwell GP, Tornambe R. Management of mammary subpectoral implant distortion. Clin Plast Surg 1988;15:60111.

Supplemental Video $\mathrm{S} 1$. Video of animation grading.

Supplemental data can be found at: https://doi.org/10.5999/aps.2017.01242.v001

Supplemental Video S2. Video of animation grading.

Supplemental data can be found at: https://doi.org/10.5999/aps.2017.01242.v002 